\title{
THE SPATIO-KINEMATIC STRUCTURE OF THE CO ENVELOPES IN EVOLVED PLANETARY NEBULAE
}

\author{
R. BACHILLER ${ }^{1}$, P.J. HUGGINS ${ }^{2}$, P. COX ${ }^{3}$, T. FORVEILLE \\ ${ }^{1}$ Centro Astronomico de Yebes (Spain), ${ }^{2}$ New York University (USA), ${ }^{3}$ Observatoire de \\ Marseille (France), ${ }^{4}$ Observatoire de Grenoble (France)
}

We report high angular resolution mapping of the CO $(J=2 \rightarrow 1$ and $1 \rightarrow 0)$ lines in three evolved planetary nebulae (PNe): NGC 6781, NGC6772, and VV 47. The $\mathrm{CO} 2 \rightarrow 1$ observations of the ring-like nebula NGC 6781 provide the most detailed map to date of the kinematic structure of a PN envelope. The data are well explained with a model consisting of a thin, clumpy, ellipsoidal shell, which is open at the ends and is expanding with a velocity proportional to distance from the star. The molecular shell of NGC 6772 appears similar, but the gas is more confined to an equatorial ring and is much more incomplete. The molecular gas in VV 47 is in two clumpy lobes, which are likely to be the only surviving molecular condensations from an earlier, more extended equatorial distribution of the same kind. The average $\mathrm{CO}$ excitation temperature of these $\mathrm{PNe}$ is found to be $>23 \mathrm{~K}$ from the $\mathrm{CO} 2 \rightarrow 1 / 1 \rightarrow 0$ line ratio, and the mass of molecular gas is estimated to be $0.1,0.02$, and 0.002 $M \odot$ in NGC $6781, \mathrm{NGC} 677^{\circ}$, and VV 47, respectively. It appears that the ring-like PNe are formed from the dissociation and ionization of neutral ellipsoidal shells; destruction of the envelope begins with the rapid ionization of the least dense polar caps, and continues until the densest molecular material at the nebular waist is fully ionized. 